RAL-TR-98-022

\title{
Features of Quark and Lepton Mixing from Differential Geometry of Curves on Surfaces
}

\author{
José BORDES \\ bordes@ evalvx.ific.uv.es \\ Dept. Fisica Teorica, Univ. de Valencia, \\ c. Dr. Moliner 50, E-46100 Burjassot (Valencia), Spain \\ CHAN Hong-Mo \\ chanhm@ @2.rl.ac.uk \\ Rutherford Appleton Laboratory, \\ Chilton, Didcot, Oxon, OX11 0QX, United Kingdom \\ Jakov PFAUDLER \\ jakov@thphys.ox.ac.uk \\ Dept. of Physics, Theoretical Physics, University of Oxford, \\ 1 Keble Road, Oxford, OX1 3NP, United Kingdom \\ TSOU Sheung Tsun \\ tsou@ maths.ox.ac.uk \\ Mathematical Institute, University of Oxford, \\ 24-29 St. Giles', Oxford, OX1 3LB, United Kingdom
}

\begin{abstract}
It is noted that the CKM matrix elements for both quarks and leptons as conceived in the Dualized Standard Model (DSM) can be interpreted as direction cosines obtained by moving the Darboux trihedron (a 3-frame) along a trajectory on a sphere traced out through changing energy scales by a 3 -vector factorized from the mass matrix. From the 'Darboux' analogues of the well-known Serret-Frenet formulae for space curves, it is seen that the corner elements $\left(V_{u b}, V_{t d}\right.$ for quarks, and $U_{e 3}, U_{\tau 1}$ for leptons) are associated with the (geodesic) torsion, while the other off-diagonal elements $\left(V_{u s}, V_{c d}\right.$ and $V_{c b}, V_{t s}$ for quarks, and $U_{e 2}, U_{\mu 1}$ and $U_{\mu 3}, U_{\tau 2}$ for leptons) with the (respectively geodesic and normal) curvatures of the trajectory. From this it follows that (i) the corner elements in both matrices are much smaller than the other elements, (ii) the $U_{\mu 3}, U_{\tau 2}$ elements for the lepton CKM matrix are much larger than their counterparts in the quark matrix. Both these conclusions are strongly borne out by experiment, for quarks in hadron decays and for leptons in neutrino oscillations, and by previous explicit calculations within the DSM scheme.
\end{abstract}


The Cabibbo-Kobayashi-Moskawa matrix which gives the relative orientation in generation space between the state vectors of the three physical states of $U$-type and $D$-type quarks has now been measured to a fair accuracy by experiment. In the latest databook [1], it is given as:

$$
|C K M|_{\text {quark }}=\left(\begin{array}{lll}
0.9745-0.9760 & 0.217-0.224 & 0.0018-0.0045 \\
0.217-0.224 & 0.9737-0.9753 & 0.036-0.042 \\
0.004-0.013 & 0.035-0.042 & 0.9991-0.9994
\end{array}\right),
$$

where the modulus sign on the left-hand side means that we are giving only the absolute values of the entries.

A similar matrix can in principle be defined between charged leptons and neutrinos, here referred to as the leptonic CKM matrix, for which the experimental information is still rather fragmentary in comparison, but some features of which are beginning to emerge. First, from the so-called muon anomaly observed in atmospheric neutrinos [2, 3, 4, 5], one obtains that the matrix element relating the muon to the heaviest neutrino $\nu_{3}$, namely $\left|U_{\mu 3}\right|$ is rather large, say roughly $0.45-0.85$, according to a recent analysis [ [6] . Secondly, from the absence of any oscillation effects in reactor experiments such as CHOOZ [7], one obtains, at least for the squared mass difference $\Delta m_{23}^{2}$ of the two heaviest neutrinos being in the range $10^{-2}-10^{-3} \mathrm{eV}^{2}$ as favoured by the atmospheric neutrino data, that the element relating the electron to $\nu_{3}$, namely $\left|U_{e 3}\right|$, is small, say roughly less than 0.15 [6]. Thirdly, for the solar neutrino puzzle [8], one is offered several solutions, either (i) the so-called long wave-length oscillation (LWO) solution which wants $\left|U_{e 2}\right| \sim 0.4-0.7$ [9, 10], or (ii) the so-called MSW solution making use of the Mikheyev-Smirnov-Wolfenstein [11, 12] mechanism which wants either $\left|U_{e 2}\right| \sim 0.4-0.6$ (large angle solution) or $\left|U_{e 2}\right| \sim 0.03-0.05$ (small angle solution) [13]. If we choose either the LWO solution or the large angle MSW solution for which there seems to be some preference, we obtain for the leptonic CKM matrix the following tentative arrangement:

$$
|C K M|_{\text {lepton }}=\left(\begin{array}{ccc}
* & 0.4-0.7 & 0.0-0.15 \\
* & * & 0.45-0.85 \\
* & * & *
\end{array}\right)
$$

In as much as the CKM matrix is a rotation matrix near to the identity, $|C K M|$ is roughly symmetric. And given that we are at present ignoring the CP-violating phase, it contains only 3 independent real parameters, so 
that from the bounds of the 3 elements specified above rough bounds for the other elements can be calculated, but we shall not need them for our present discussion.

Comparing the two matrices (11) and (21), one notices the following salient features. First, the corner elements 13 and 31 are in both cases much smaller than the rest. Second, the 23 element of the lepton CKM matrix is much larger than its counterpart in the quark CKM matrix. Third, the 12 (21) elements for both matrices are sizeable and of roughly the same magnitude. Now these features are highly significant in physical terms, for the quark case in explaining the branching ratios of various hadronic decays, and for the lepton case, as outlined above, in explaining certain effects in neutrino oscillations. It would thus be important to understand how these features arise in any attempt at modelling quark and lepton mixing.

Now we have recently suggested a scheme based on the dual properties of the Standard Model (DSM) [14] which purports to explain the existence of exactly three generations as spontaneously broken dual colour and affords at the same time a means for evaluating perturbatively the CKM matrix for both the quark [15] and the lepton [16] case. Calculations to 1-loop level have already been performed giving quite satisfactory answers which have the empirical features emphasized in the above paragraph. It would thus be interesting to find the theoretical reason within the scheme why these particular features should emerge.

Let us recall some basic properties of the fermion mass matrix in the DSM scheme. By virtue of the manner in which the generation (dual colour) symmetry is broken, as suggested by the Higgs fields occurring naturally in the theory, the fermion mass matrix in the DSM is factorizable at tree-level, and even remains so after loop corrections [14]. Thus,

$$
m^{\prime}=m_{T}\left(\begin{array}{l}
x^{\prime} \\
y^{\prime} \\
z^{\prime}
\end{array}\right)\left(x^{\prime}, y^{\prime}, z^{\prime}\right) .
$$

Apart from the mass scale $m_{T}$ which may be taken to be the mass of the highest state of the fermion-type under consideration, the whole information of the mass matrix is encoded in the vector $\left(x^{\prime}, y^{\prime}, z^{\prime}\right)$. This vector rotates with changing energy scales, thus tracing out a trajectory in generation space, and in the approximation where one neglects the scale-dependence of the length of $\left(x^{\prime}, y^{\prime}, z^{\prime}\right)$ which one cannot compute at present, one can normalize 
the vector thus:

$$
x^{2}+y^{\prime 2}+z^{\prime 2}=1,
$$

so that the trajectory lies on the unit sphere. Further, without loss of generality we can take $x^{\prime} \geq y^{\prime} \geq z^{\prime}$.

Although the trajectory traced out by $\left(x^{\prime}, y^{\prime}, z^{\prime}\right)$ can in principle be different for different fermion-types it was found in [15] by fitting experiment that, to a very good accuracy, all fermion-types run on the same trajectory with the same speed with respect to the energy scale $\mu$, only differing by the locations of the physical states. The actual trajectory obtained in [15] together with the locations on it of the 12 physical states [15, 16] are shown in Figure 1 .

We shall not detail here the criterion used in the DSM for determining the location, namely the mass, of the lower generation states, since it is of no great relevance for our present discussion, but refer the reader to [15]. What matters for us here is how the state vectors in generation (i.e. dual colour) space of the physical states are defined.

First, for each fermion-type (i.e. whether $U$ for $U$-type quarks, $D$ for $D$ type quarks, $L$ for charged leptons or $N$ for neutrinos), the highest generation state (i.e. $t, b, \tau, \nu_{3}$ in the above order) is defined as that eigenstate $\left|\mathbf{v}_{1}\right\rangle$ of the mass matrix $m^{\prime}$ in (3) with non-zero eigenvalue, taken at the scale equal to the mass of that generation, namely at $\mu=m_{t}, m_{b}, m_{\tau}, m_{\nu_{3}}$ respectively. It is easily seen that in Figure 11 these are just the radial vectors $\left(x^{\prime}, y^{\prime}, z^{\prime}\right)$ themselves taken at the appropriate locations.

Secondly, the state vector of the second generation which we called $\left|\mathbf{v}_{2}^{\prime}\right\rangle$ in [15] is defined again as the eigenvector with non-zero eigenvalue but now of the submatrix of $m^{\prime}$ in the 2-dimensional subspace orthogonal to $\left|\mathbf{v}_{1}\right\rangle$ evaluated at the scale equal to the second generation mass. At the location on the trajectory occupied by the second generation, the vector $\left(x^{\prime}, y^{\prime}, z^{\prime}\right)$ would have rotated to a different direction, say $\left|\tilde{\mathbf{v}}_{1}\right\rangle$, as illustrated in Figure 2. The vector $\left|\mathbf{v}_{2}^{\prime}\right\rangle$ is the vector orthogonal to $\left|\mathbf{v}_{1}\right\rangle$ which soaks up all the 'leakage' from this rotation. It is easy to see then that $\left|\mathbf{v}_{2}^{\prime}\right\rangle$ lies on the plane formed by $\left|\mathbf{v}_{1}\right\rangle$ and $\left|\tilde{\mathbf{v}}_{1}\right\rangle$ as shown in Figure 2. Obviously, the state vector $\left|\mathbf{v}_{3}^{\prime}\right\rangle$ of the lowest generation is then, as also shown in the figure, the vector orthogonal to both $\left|\mathbf{v}_{1}\right\rangle$ and $\left|\mathbf{v}_{2}^{\prime}\right\rangle$.

Imagine now that the locations of the highest two generations are rather close together, which is indeed the case for all the 4 fermion-types shown in Figure 11. In that case, the vector $\left|\mathbf{v}_{2}^{\prime}\right\rangle$ approaches the tangent vector of the trajectory at $\left(x^{\prime}, y^{\prime}, z^{\prime}\right)$. In other words, in this approximation, the 3 state 


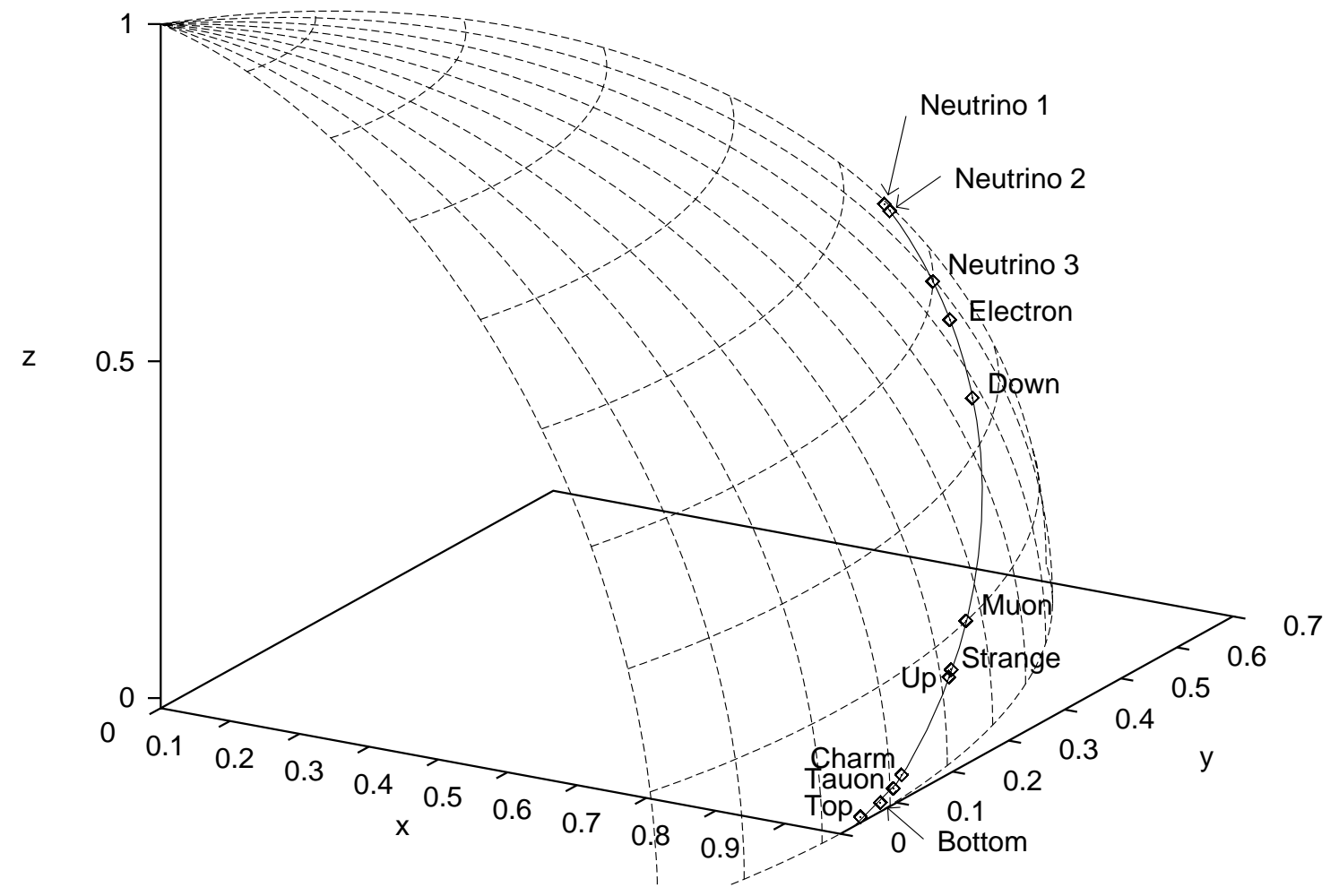

Figure 1: The trajectory traced out by $\left(x^{\prime}, y^{\prime}, z^{\prime}\right)$ and the locations on it of the 12 physical fermion states.

vectors for the 3 generations coincide with the triad formed by the radial vector, the tangent vector to the trajectory, and the normal vector to both.

Next, still in this approximation, consider the triad of state vectors for a pair of fermion-types, i.e. either $U$ - with $D$-type quarks, or the charged leptons $L$ with the neutrinos $N$. At the location (of the combined two highest generations) of each fermion-type, we have associated a triad of state vectors as depicted in Figure 3. The triad at one location is obtained just by transporting the triad from the other location along the trajectory on the sphere. Now the CKM matrix is by definition the matrix giving the relative orientation of the triad of physical state vectors between the $U$-type and $D$ - 


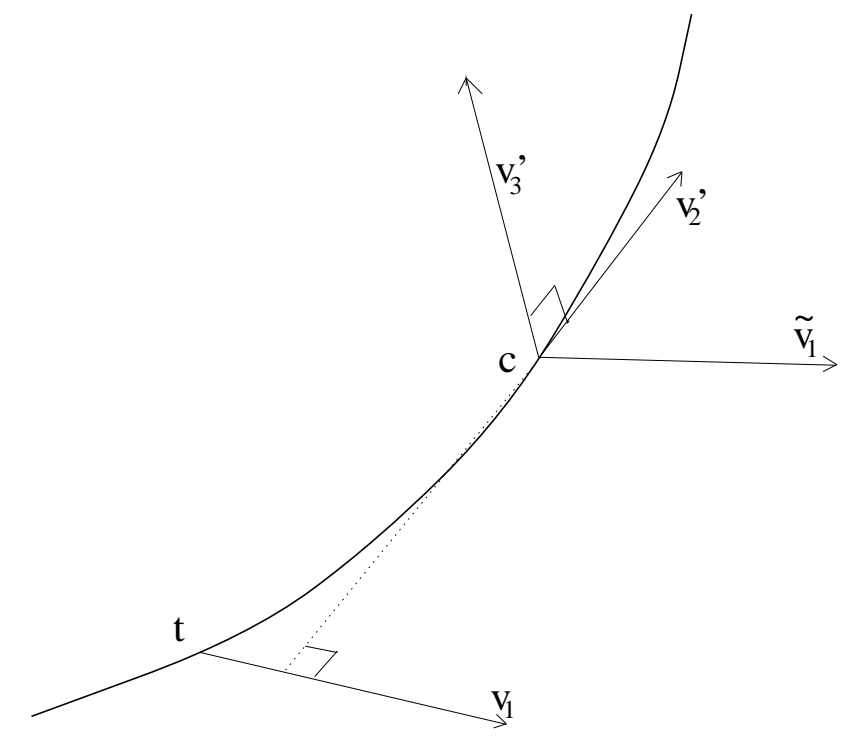

Figure 2: The state vectors of the 3 physical states belonging to the 3 generations of each fermion-type.

type quarks, or in the leptonic version between the charge leptons and the neutrinos. In view of our observations above, we conclude that it can be written as:

$$
C K M=P_{s} \exp \int_{U}^{D} d s A(s),
$$

similar in form to the Dirac phase factor in gauge theory language, where $s(\mu)$ is the distance on the trajectory, $\mu$ is the energy scale, and $A$ is some rotation matrix yet to be specified. Notice that to 1-loop order, the distance $s$ travelled per unit change in the energy scale $\mu$ is proportional to the Yukawa coupling strength squared $\rho^{2}$, which therefore plays the role of the gauge coupling in the usual formula for the Dirac phase factor in gauge theory.

To specify the matrix $A$, we let the two locations corresponding to the two fermion-types approach each other on the trajectory and evaluate the CKM matrix to first order in the distance $\Delta s$ between the two locations. This can easily be done using some elementary results from the differential geometry of curves on surfaces [17]. Let $\Gamma$ be a curve parametrized by arc-length $s$ lying on a surface $S$, in our case the unit sphere. At every point on the curve $\Gamma$, we have $\mathbf{N}$ the unit normal to the surface, $\mathbf{T}$ the unit tangent to the curve, and we can define a third unit vector $\mathbf{B}=\mathbf{N} \wedge \mathbf{T}$ normal to both. These form what is sometimes called the Darboux trihedron and are actually exactly the triad we are interested in, since for the sphere the radial and normal vectors coincide. Differentiating these vectors with respect to arc-length we get the following relations which we may call the Serret-Frenet-Darboux formulae 


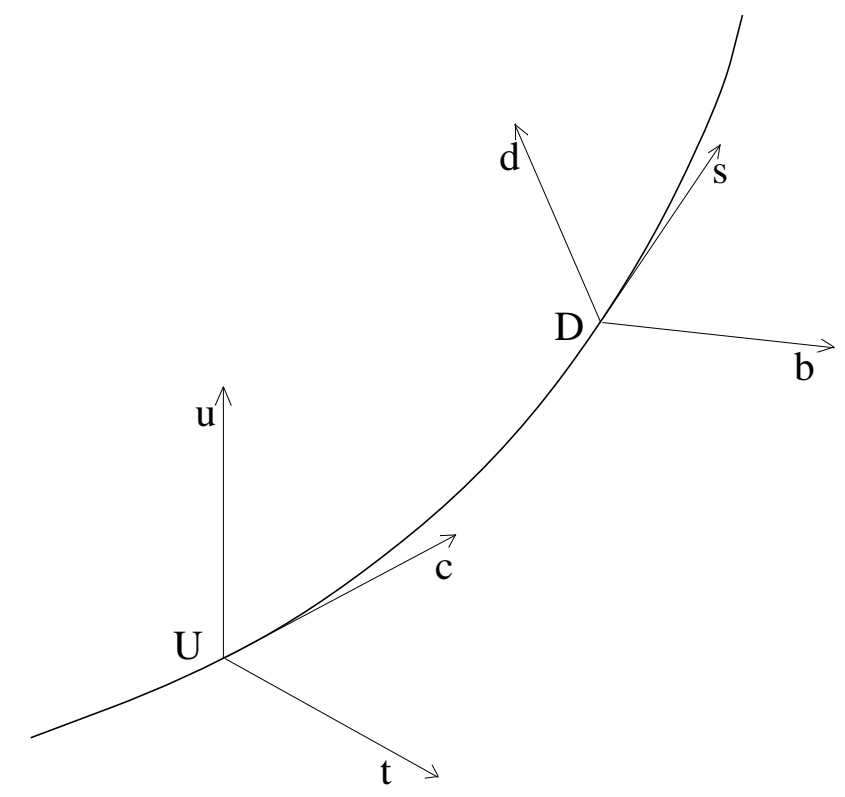

Figure 3: Two triads of state vectors for two fermion-types transported along a common trajectory.

in analogy to the usual Serret-Frenet formulae for space curves:

$$
\begin{aligned}
\mathbf{N}^{\prime} & =-\kappa_{n} \mathbf{T}-\tau_{g} \mathbf{B}, \\
\mathbf{T}^{\prime} & =\kappa_{g} \mathbf{B}+\kappa_{n} \mathbf{N}, \\
\mathbf{B}^{\prime} & =-\tau_{g} \mathbf{N}-\kappa_{g} \mathbf{T} .
\end{aligned}
$$

Here $\kappa_{g}$ is the geodesic curvature, $\kappa_{n}$ the normal curvature, and $\tau_{g}$ the geodesic torsion of the curve $\Gamma$ on $S$. Recall that the Serret-Frenet formula for $\mathbf{T}^{\prime}$ defines both the curvature $\kappa$ of the curve and its normal $\mathbf{n}$ at the given point:

$$
\mathbf{T}^{\prime}=\kappa \mathbf{n},
$$

and that, being normal to $\mathbf{T}$, the curvature vector $\kappa \mathbf{n}$ lies on the $\mathbf{N}-\mathbf{B}$ plane. The second equation in (6) gives its decomposition along $\mathbf{B}$ and N. Arranged in the conventional order for physicists in the form of a CKM matrix the formulae in (6) read as:

$$
C K M \sim\left(\begin{array}{ccc}
1 & -\kappa_{g} \Delta s & -\tau_{g} \Delta s \\
\kappa_{g} \Delta s & 1 & \kappa_{n} \Delta s \\
\tau_{g} \Delta s & -\kappa_{n} \Delta s & 1
\end{array}\right)
$$

\footnotetext{
${ }^{1}$ Notice that it is conventional to label the rows and columns of the CKM matrix in order of increasing mass, which is opposite to that adopted above, following [15], for labelling the state vectors $\left|\mathbf{v}_{i}^{\prime}\right\rangle$ of the three generations.
} 
to first order in $\Delta s$, this being the distance between the locations for the two fermion-types. This gives the matrix $A$ in (5) as:

$$
A=\left(\begin{array}{ccc}
0 & -\kappa_{g} & -\tau_{g} \\
\kappa_{g} & 0 & \kappa_{n} \\
\tau_{g} & -\kappa_{n} & 0
\end{array}\right) .
$$

Suppose now that the separation in locations between the two-fermion types on the trajectory is actually small as is indeed the case for the $U$ - and $D$-type quarks, as seen in Figure 11. The CKM matrix is then to a good approximation given just by (8) above. We notice first that the off-diagonal corner elements of the matrix are proportional to the geodesic torsion $\tau_{g}$ while the other off-diagonal elements are proportional to the curvatures $\kappa_{g}$ and $\kappa_{n}$. For the particular case of a curve on a sphere, which is our case, the geodesic torsion $\tau_{g}$ vanishes [17], so that the corner elements of the CKM matrix are of second order in $\Delta s$ and therefore relatively small. Comparing this with the empirical CKM matrix for quarks quoted in (1), one sees that this prediction is very clearly borne out. Secondly, the elements $V_{c b}$ and $V_{t s}$ are proportional to the normal curvature $\kappa_{n}$ which for the sphere is constant, independent either of the trajectory or of the location. It follows then from (8) that their values are approximately given just by the distance, measured in units of the radius of the sphere, between the locations of $t$ and $b$ on the trajectory. From Figure 1, one estimates this to be of order of a few percent. Again, the prediction is seen to be borne out in (1). Both these special features are also what one finds in the explicit calculation carried out in [15]. As for the remaining off-diagonal elements $V_{u s}$ and $V_{c d}$, namely the Cabibbo angle, it is in a sense a little special. Being proportional to the geodesic curvature $\kappa_{g}$ which depends both on the location and on the trajectory, it can be sizeable even for small separation. This is seen to be indeed the case in the empirical matrix (11) and agrees with the fact that in the calculation [15], the Cabibbo angle can be fitted to the experimental value by choosing the trajectory.

What would happen in the case of the leptons where the separation between the two fermion-types, i.e. between $\tau$ and $\nu_{3}$, is not so small, as seen in Figure 11? We shall need in that case to evaluate the 'Dirac phase factor' (5) above, as was done numerically in [16]. However, even without an explicit calculation we can already draw some qualitative conclusions. First, the corner element $U_{e 3}$ being of second order in the distance travelled will remain small. This checks with the empirical estimates quoted in (2) and with the calculation [16]. Secondly, the normal curvature $\kappa_{n}$ being constant 
all along the trajectory, its contribution to the element $U_{\mu 3}$ will accumulate over the larger distance here and become much larger than its counterpart $V_{c b}$ in the quark case. In fact, if one just crudely takes the formula (8) and assumes that the element is still roughly proportional to the distance travelled from $\tau$ to $\nu_{3}$, one obtains from Figure 1 that $U_{\mu 3}$ would be some 20 times larger than $V_{c b}$ in the quark matrix, which estimate is seen already to be in fair agreement both with the empirical matrices in (1) and (2), and with the explicit calculations in [15, 16]. Thirdly, in contrast, the remaining off-diagonal element $U_{e 2}$ depends on the geodesic curvature $\kappa_{g}$ which varies with the location. Its value therefore cannot be estimated from just the separation between the locations of the two fermion-types. There is thus no reason to expect a value for it very different in magnitude from the values of $V_{u s}$ or $V_{c d}$ in the quark case, nor are the values as seen in (1) and (2) very different empirically.

There is a further qualitative conclusion that one can draw from the preceding analysis about the CKM matrix which, however, cannot be checked against experiment but only against the calculation in [15]. As mentioned above, the normal curvature $\kappa_{n}$ on a sphere does not depend on the trajectory. Hence, the elements $V_{c b}$ and $V_{t s}$, being to first order in $\Delta s$ proportional to the normal curvature $\kappa_{n}$, is also expected to be approximately independent of the trajectory. We then see why we were able, when fitting the CKM matrix in [15], first to fit the elements $V_{c b}$ and $V_{t s}$ by adjusting the Higgs vev $y$, and then to fit the rest by adjusting the other vev $z$, for the first two elements will remain roughly constant in changing $z$ which has the effect mainly of changing the trajectory.

The above analysis was done in the approximations (a) that the locations on the trajectory of the two highest states of each fermion-type are close together, and (b) that the vector $\left(x^{\prime}, y^{\prime}, z^{\prime}\right)$ has a normalization independent of the energy scale $\mu$ and hence moves on a sphere. However, the qualitative features deduced above for the quark and lepton CKM matrices will still remain even if one relaxes these approximations so long as the scale-dependence of the normalization of $\left(x^{\prime}, y^{\prime}, z^{\prime}\right)$ is not too strong. In any case, the CKM matrix can always be regarded as a 'Dirac phase factor' of the form (5), only in general the matrix $A$ appearing there will be more involved.

Apart from the elegance in giving us a geometrical significance for the CKM matrix, and a qualitative understanding of the values of its elements in simple geometrical terms, the above observations have the practical value, we think, in giving us also a nontrivial check on the basic structure of the 
DSM scheme. The fact that the three off-diagonal elements 13, 23, and 12 are predicted to have quite different salient features all agreeing with experiment, depends crucially on the assignment in the scheme of the highest, middle and lowest generation states to respectively the radial vector, the tangent vector and the vector normal to both, on the trajectory. And this trajectory is traced out by the vector $\left(x^{\prime}, y^{\prime}, z^{\prime}\right)$, which is in turn obtained from a factorizable mass matrix. Quite a number of structural details of the scheme are thus involved in yielding these predictions. Further, the fact that rough values of the empirical matrix elements can already be deduced just from the DarbouxSerret-Frenet formulae (6) makes the agreement with experiment obtained in [15] and [16] much less likely to be mere accidents of the calculations.

\section{Acknowledgement}

One of us (JB) acknowledges support from the Spanish Government on contract no. CICYT AEN 97-1718, while another (JP) is grateful to the Studienstiftung d.d. Volkes and the Burton Senior Scholarship of Oriel College, Oxford for financial support.

\section{References}

[1] Particle Physics data booklet, (1996), from R.M. Barrett et al., Phys. Rev. D54, 1, (1996). See also the updates on the PDG's website (http://pdg.lbl.gov/).

[2] K.S. Hirata et al., Phys. Letters B205, 416 (1988); B280, 146 (1992); Y. Fukuda et al. Phys. Letters B335, 237 (1994).

[3] Superkamiokande Collaboration, as reported by Y. Totsuka at XVIII International Symposium on Lepton-Photon Interactions, July (1997) Hamburg, Germany, to appear in the proceedings (http://www.desy.de/lp97-docs/proceedings/lp22/lp22.ps); E. Kearns, talk at News about SNU, ITP Workshop, Santa Barbara, Dec. 1997 (http://www.itp.ucsb.edu/online/snu/kearns/oh/all.html).

[4] D. Casper et al., Phys. Rev. Letters 66, 2561, (1991); R. Becker-Szendy et al., Phys. Rev. D46, 3720, (1992); Nucl. Phys. B (Proc. Suppl.) 38, 331, (1995). 
[5] T. Kafka, Nucl. Phys. B (Proc. Suppl.) 35, 427, (1994); M. Goodman, ibid 38, 337, (1995); W.W.M. Allison et al., Phys. Letters B391, 491, (1997).

[6] C. Giunti, C.W. Kim, and M. Monteno, hep-ph/9709439 (1997).

[7] CHOOZ collaboration, M. Apollonio et al., hep-ex/9711002, (1997).

[8] See e.g. T. Kuo and J. Pantaleone, Rev. Mod. Phys. 61, 1537 (1988).

[9] V. Barger, R.J.N. Phillips, and K. Whisnant, Phys. Rev. Letters 69, 3135, (1992).

[10] P.I. Krastev and S.T. Petcov, Phys. Rev. Letters 72, 1960 (1994).

[11] L. Wolfenstein, Phys. Rev. D17, 2369, (1978).

[12] S.P. Mikheyev and A. Yu. Smirnov, Nuovo Cimento 9C, 17, (1986).

[13] For a recent analysis, see e.g. G.L. Foglio, E. Lisi, and D. Montanino, Phys. Rev. D54, 2048, (1996).

[14] Chan Hong-Mo and Tsou Sheung Tsun, hep-th/9701120, Phys. Rev. D57, 2507, (1998).

[15] J. Bordes, Chan Hong-Mo, J. Faridani, J. Pfaudler, and Tsou Sheung Tsun, RAL-TR-97-068, hep-ph/9712276, (1997).

[16] J. Bordes, Chan Hong-Mo, J. Pfaudler, and Tsou Sheung Tsun, RALTR-98-021, hep-ph/9802420, (1998).

[17] See e.g. L.P. Eisenhart, A Treatise on the Differential Geometry of Curves and Surfaces, Ginn and Company 1909, Boston; M.P. do Carmo, Differential Geometry of Curves and Surfaces, Prentice-Hall 1976, Englewood Cliffs, New Jersey. 